\title{
FAINT SURFACE PHOTOMETRY OF THE HALO OF M31
}

\author{
CHRIS PRITCHET
}

Department of Physics and Astronomy, University of Victoria, P.O. Box 3055, Victoria, BC, Canada V8W 3P6

\author{
AND \\ SIDNEY VAN DEN BERGH \\ Dominion Astrophysical Observatory, 5071 W. Saanich Road, \\ Victoria, BC, Canada V8X $4 M 6$
}

\begin{abstract}
We have obtained counts of stars near the tip of the red giant branch of M31, and have used these counts to estimate the surface brightness of the halo of M31 down to a level of $\mu_{V} \simeq 30 \mathrm{mag} \operatorname{arcsec}^{-2}$ $(R \approx 20 \mathrm{kpc})$. The surface brightness along the minor axis of the M31 halo is well-represented by a single de Vaucouleurs law $(0.2 \lesssim R[\mathrm{kpc}] \lesssim 20)$. Alternatively, the outer halo of M31 can also be modelled by a power-law density distribution of the form $\rho(R) \propto R^{-5}$. This result suggests that the globular cluster component of the halo of M31 (for which $\rho \propto R^{-3}$ ) is more extended than the stellar halo of this galaxy. At $\mu_{V} \approx 28 \mathrm{mag}$ $\operatorname{arcsec}^{-2}(R \approx 10 \mathrm{kpc})$, the axial ratio of the halo of M31 is found to be $c / a=0.55 \pm 0.05$.
\end{abstract}

\section{Introduction}

A paper on M31 might seem a somewhat odd choice for a meeting on the Milky Way. Yet very little is known about the structure of the Milky Way stellar halo, primarily because we are immersed in it, and are locally swamped by stars belonging to the disk. It therefore seems reasonable to see what can be learned about stellar halos by studying other galaxies, in particular M31, which is close enough that its stellar halo can be resolved.

In this paper we use a method involving counting individual stars to determine the surface brightness distribution of the halo of M31. Surface brightness levels $\mu_{V} \approx 30 \mathrm{mag} \operatorname{arcsec}^{-2}$ are reached; we predict that $\sim 32$ 
mag $\operatorname{arcsec}^{-2}$ is within reach of future observations. Further details of this work can be found in Pritchet and van den Bergh (1994, hereafter PV94).

\section{From Star Counts to Surface Brightness}

Observations were obtained with the Canada-France-Hawaii $3.6 \mathrm{~m}$ Telescope, in conjunction with CCD detectors with field size $2 ! 2 \times 3 ! 3$. Total exposures were $\sim 1 \mathrm{hr}$ through $B$ and $V$ filters. Seeing was typically $\sim 1^{\prime \prime}$ FWHM for all exposures.

Most of the program fields were chosen to lie along the minor axis of M31 (fields M0-M5, $40^{\prime}-5^{\circ}$ ); two fields (E1 and E2) were observed at position angle between the major and minor axes, and three fields (R1-R3) were observed at large distances $\left(\sim 10^{\circ}\right)$ from M31 as "reference" fields, to monitor foreground and background contamination. The data were analyzed using DAOPHOT (Stetson 1987). Completeness fractions and photometric errors were evaluated with artificial star experiments (typically 5 runs of 100 added stars for each field and bandpass). Image moments (Kron 1980) were used to find and reject extended objects.

Luminosity distributions in the $V$ band were computed by counting each star as $1 / f\left(m_{i}\right)$, where $f$ is the completeness fraction at $m_{i}$, the magnitude of star $i$. Examples of some luminosity distributions can be found in PV94. Our analysis of the surface brightness of the fields is confined to the magnitude range $22<V<24$, and avoids both unnecessary contamination by field stars at the bright end, and severe incompleteness problems at the faint end (since $50 \%$ completeness is at $V=24.5$ ).

The true surface brightness in each field is determined by (1) subtracting the contribution of contaminants in the magnitude range $22<V<24$, and (2) correcting for the missed light from M31 halo stars outside the magnitude range $22<V<24$. Correction (1) is $\mu_{V}=30.26 \pm 0.11 \mathrm{mag} \mathrm{arcsec}^{-2}$ for the contaminant luminosity distribution that was derived above. Correction (2) depends on metallicity, and is about $-1.64 \mathrm{mag}$ (appropriate to a 47 Tuc metallicity - cf. Mould and Kristian 1986, Pritchet and van den Bergh 1988, Christian and Heasley 1991, van den Bergh and Pritchet 1992). The reader is referred to PV94 for further details on the above corrections.

\section{Luminosity Profile and Flattening of M31 Halo}

Fig. 1 shows our observed surface brightness data for the M31 halo; filled squares represent minor axis data, and open squares represent fields E1 and E2 (between major and minor axis - to be discussed below). Also shown in Fig. 1 are data from Walterbos and Kennicutt (1987). Our observations join smoothly onto an extrapolation of these data, and also onto the data of de Vaucouleurs (1958). 


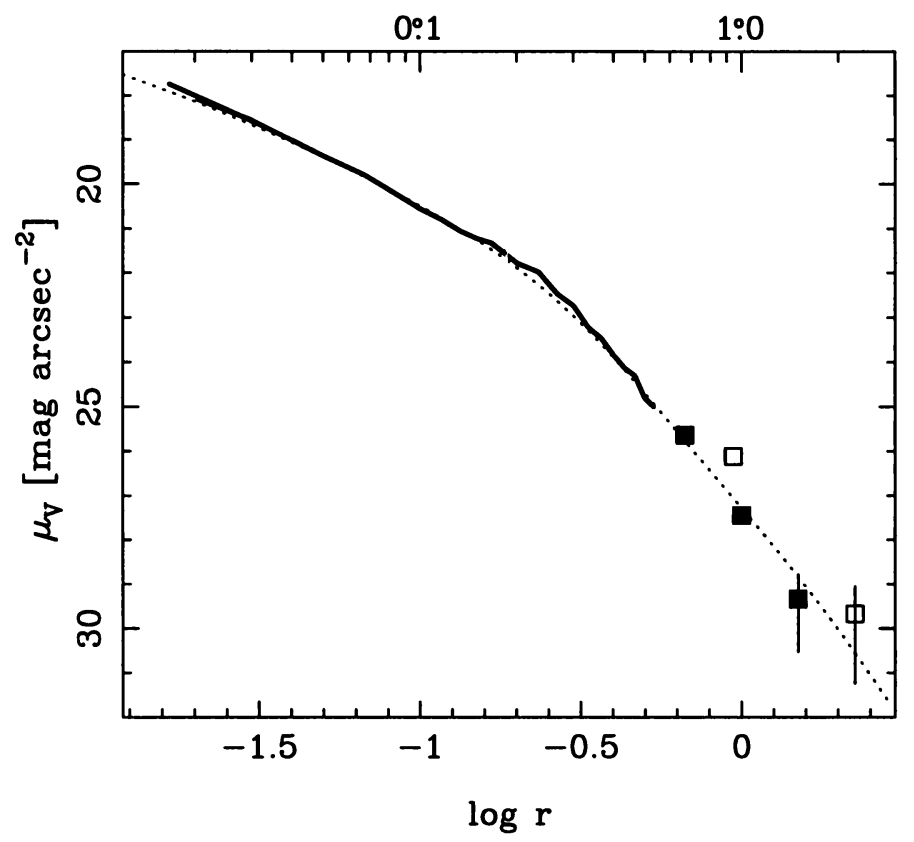

Figure 1. Surface photometry of M31 plotted against angle (degrees) from the centre of M31. The solid squares represent minor axis photometry, and the heavy solid line represents minor axis photometry of Walterbos and Kennicutt (1987). The dotted line represents a sum of the disk plus $r^{\frac{1}{4}}$ spheroid model. The open squares refer to our fields that are between the major and minor axis of M31. These latter fields are brighter than fields on the minor axis, indicating that the halo is flattened.

The disk contribution is estimated from Walterbos and Kennicutt (1988, WK88) to be quite small in Fig. 1 ; it is at most $40 \%$ of the light at about $15^{\prime}$, but drops to less than $10 \%$ for our outer fields. The dotted curve in Fig. 1 shows the sum of the disk plus de Vaucouleurs (dV) law spheroid contributions from the WK88 model. This model provides an excellent fit to the data plotted in Fig. 1. It also provides a reasonable fit to the data of Kent (1983) and WK88 at smaller radii ( at least for $r \gtrsim 1^{\prime}$ ).

We conclude that a single de Vaucouleurs law luminosity profile (with minor axis effective radius $b_{\text {eff }} \simeq 1.3 \mathrm{kpc}$ ) can fit the spheroid of M31 from the inner bulge $\left(r \approx 1^{\prime}, R \simeq 200 p c\right)$ all the way out to the halo $(r \gtrsim 1: 5, R \gtrsim 20 \mathrm{kpc})$. The behavior of the luminosity profile within $1^{\prime}$ is much more complicated (e.g., Light, Danielson, and Schwarzschild 1974; Kent 1983).

A single power law cannot represent the run of surface brightness in Fig. 
1. The outer minor axis observation require a power-law in projected surface brightness $I \propto r^{-4}$, or a density profile $\rho \propto R^{-5}$. These $\rho(R)$ distributions are clearly much steeper than the $\rho \propto R^{-2.5}$ observed at $r<0$ :1, and also steeper than the $\rho \propto R^{-3.5}$ observed for the Milky Way.

Two of our fields (E1 and E2) are situated well away from the minor axis of $\mathrm{M} 31$, at position angles between $30^{\circ}$ and $45^{\circ}$. Fig. 1 shows that these fields possess an excess of light relative to minor axis fields at the same radial distance from the center of $\mathrm{M} 31$. This clearly demonstrates that the halo of M31 is flattened, with $c / a=0.55 \pm 0.05$ (after correcting for disk light).

\section{Comparison of M31 with the Milky Way and Other Galaxies}

How does the minor axis profile of M31 compare with that of the Milky Way? To answer this question, we consider surface photometry of the bulge of the Milky Way by de Vaucouleurs and Pence (1975). They showed the bulge of the MW is well-fitted by a de Vaucouleurs law; however, extrapolating this $\mathrm{dV}$ law to the Galactic poles overestimates the known surface brightness from halo stars by more than 2 mag! A similar effect is seen for NGC 4565: the bulge of this galaxy is much brighter than would be predicted by extrapolating the halo light profile (van der Kruit and Searle 1981a). On the other hand, the halo of the edge-on Sbc galaxy NGC 891 (which resembles the MW quite closely) obeys an $r^{\frac{1}{4}}$ law from 1.5 to $8 \mathrm{kpc}$ (van der Kruit and Searle 1981b). The situation with the halos/bulges of other galaxies is confusing: some do, and some don't, follow a single dV law (PV94). This seems to suggest (but does not prove) that (i) bulge and halo formation is decoupled; and (ii) the continuity of the bulge and halo light in M31 may be an accident. It should be noted that M31 now possesses the best surface photometry of any bulge/halo system, so that a comparison with other galaxies is not easy.

Expressing the halo density law as a power-law $\rho(r) \propto r^{n}$, we find an exponent $n$ approaching -5 ; this is very different from the values of $n=-3$ to -3.5 that are often quoted for the Milky Way. Now, it seems unlikely that M31 would have a shallower power-law density drop-off than the Milky Way, given its earlier morphological type (which folklore dictates should imply a more prominent spheroidal population). Nor do we consider the canonical MW power-law values of $n$ to be reliable, since they are mostly based on globular clusters (which do not necessarily follow the density law of the stellar halo in other galaxies), or RR Lyrae stars (whose density law may be strongly modulated by population dependent [second parameter] effects). Thus we consider it to be entirely possible that the MW stellar halo may follow a density law as steep as that implied for M31. Given the 
steepness of the implied exponent $(n \simeq-5)$, this would have important ramifications for the spatial extent of the MW halo. Clearly it would be of enormous interest to obtain better estimates of the run of density in the MW stellar halo based on subdwarfs.

The flattening $c / a$ that we have obtained for the M31 halo is quite comparable to that observed for other $\mathrm{g}$ ' laxies. NGC 891 possesses $c / a \approx$ 0.5 , with a considerable dependence on radius (van der Kruit and Searle $1981 b$ ). The halo of NGC 4565 may be even more flattened, reaching $c / a \simeq$ 0.4 (van der Kruit and Searle 1981a). On the other hand, the earlier type (Sab) galaxy NGC 7814 has $c / a \simeq 0.57$ at $\mu_{J} \lesssim 26 \mathrm{mag} \operatorname{arcsec}^{-2}$, with little or no radial variation (van der Kruit and Searle 1982). This latter result is more similar to that which is observed for M31, and also for the Milky Way (e.g., Wyse and Gilmore 1989 and references therein).

The globular cluster system of the M31 halo has a density profile $\rho \propto$ $R^{-3}$ (Racine 1991), identical to that observed for Milky Way globulars (Racine and Harris 1989). This should be compared with the density profile $\rho \propto R^{-5}$ observed for M31 halo stars at $R \gtrsim 10 \mathrm{kpc}$, or with $\rho \propto R^{-4}$ at smaller radius. We conclude that the globular cluster system of M31 is more extended than the stellar halo of this galaxy. This conclusion is confirmed by the work of Fusi Pecci et al. (1993), who show that the M31 globular cluster system follows an $r^{\frac{1}{4}}$ law in projected density, with effective radius $r_{\text {eff }}=4.6 \mathrm{kpc}$. The fact that this value of $r_{\text {eff }}$ is significantly larger than the value of $2.0 \mathrm{kpc}$ measured for the stellar halo ( $\$ 4)$ again suggests that the M31 globular cluster system is more extended than its stellar halo.

A similar effect has been noted for several well-observed giant ellipticals in the Virgo cluster (e.g., Harris and Racine 1979; Forte, Strom, and Strom 1981; McLaughlin, Harris, and Hanes 1993). This observation has been used as an argument in favor of the view that the formation of globular clusters predates the formation of the field spheroidal population in galaxies. Although not all galaxies show this effect, it appears that most well-observed galaxies in fact have different globular cluster and stellar halo density distributions (cf. Harris 1991).

\section{Conclusions}

We have traced the halo of M31 out to a surface brightness level $~ 30$ mag $\operatorname{arcsec}^{-2}$ in $V$, corresponding to a radial distance of $\sim 20 \mathrm{kpc}$ from the center of M31. The spheroid (bulge plus halo) of M31 is well-described by a single de Vaucouleurs law, with constant (or slightly decreasing) $c / a$, from $r \simeq 1^{\prime}$ all the way out to the limit of our observations at $r \simeq 1: 5$. The axial ratio of the M31 halo is $c / a \simeq 0.55$ at $\mu_{V} \simeq 28 \mathrm{mag} \mathrm{arcsec}{ }^{-2}$. There is a hint of a possible cutoff in the surface brightness distribution beyond 
$20 \mathrm{kpc}$.

This work was supported by the Natural Sciences and Engineering Research Council of Canada.

\section{References}

Christian, C.A., \& Heasley, J.N.; 1991, AJ, 101, 848.

Forte, J.C., Strom, S.E., \& Strom, K.M.; 1981, ApJL, 245, L9.

Fusi Pecci, F., Cacciari, C., Frederici, L., \& Pasquali, A.; 1993, in The Globular Cluster Connection, ed. G.H. Smith and J.P. Brodie, A.S.P. Conference Series (San Francisco: ASP), p. 410.

Harris, W.E.; 1991, ARA\&A, 29, 543.

Harris, W.E., \& Racine, R.; 1979, ARA\&A, 17, 241.

Kent, S.M.; 1983, ApJ, 266, 562.

Kron, R.G.; 1980, ApJS, 43, 305.

Light, E.S., Danielson, R.E., \& Schwarzschild, M.; 1974, ApJ, 194, 257.

McLaughlin, D.E., Harris, W.E., \& Hanes, D.A.; 1993, ApJ, in press.

Mould, J., \& Kristian, J.; 1986, ApJ, 305, 591.

Pritchet, C.J. \& van den Bergh, S.; 1988, ApJ, 331, 135.

Pritchet, C.J. \& van den Bergh, S.; 1994, AJ, 107, 1730 (PV94).

Racine, R.; 1991, AJ, 101, 865.

Racine, R., \& Harris, W.E.; 1989, AJ, 98, 1609.

Stetson, P.B.; 1987, PASP, 99, 191.

van den Bergh, S., \& Pritchet, C.J.; 1992, in The Stellar Populations of Galaxies, ed. B.

Barbuy \& A. Renzini (Dordrecht: Reidel), p. 161.

van der Kruit, P.C., \& Searle, L.; 1981a, A\&A, 95, 105.

van der Kruit, P.C., \& Searle, L.; 1981b, A\&A, 95, 116.

van der Kruit, P.C., \& Searle, L.; 1982, A\&A, 110, 79.

Vaucouleurs, G. de; 1958, ApJ, 128, 465.

Vaucouleurs, G. de, \& Pence, W.D. 1978, AJ, 83, 1163

Walterbos, R.A.M., \& Kennicutt, R.C.; 1987, A\&ASuppl, 69, 311.

Walterbos, R.A.M., \& Kennicutt, R.C.; 1988, A\&A, 198, 61 (WK88).

Walterbos, R.A.M., \& Schwering, P.B.W.; 1987, A\&A, 180, 27.

Wyse, R.F.G., \& Gilmore, G.; 1989, Comm. Ap., 13, 135.

\section{DISCUSSION}

I. King: These are fascinating results, but I would like to remind everyone here that this paper is not related to the preceding ones. It has become a firm astronomical practice to use the word "halo" to refer to two completely distinct components of the Milky Way (or of other galaxies): the stellar halo and the dark halo, which have completely different spatial distributions.

Pritchet: Good point. I emphasize that our work refers to the stellar halo.

A. Gould: The last point at $18 \mathrm{kpc}$ seems very insecure. Could the radial profile be even steeper? 
Pritchet: Yes. The detection is clear but the value is uncertain. The profile could be steeper.

B. Burke: King mentioned that there were two kinds of halo, but there is a third halo, the high-energy halo, observed at meter wavelengths. The origin of the high-energy halo, and its dynamical behavior, are still not clear, but it probably is generated by supernova remnants bubbling up from the plane? After that, its behavior should be governed by the gravitational field in the halo. The system looks more flattened than the 2:1 flattening mentioned here- perhaps $4: 1$, but in view of our present lack of knowledge, one probably can only conclude that it supports the notion of flattened gravitational isopotentials in the halo.

Pritchet: What is the radial dependence?

(reply by Burke): Most, but not all, of the radiation is within $\mathrm{R}_{o}$. 